\title{
Focusing on medications that increase the risk of falls in older adults
}

\author{
Shelley A. Sternberg ${ }^{1}$ Paula A. Rochon ${ }^{2,3}$. Jerry H. Gurwitz ${ }^{4}$
}

Published online: 6 May 2021

(c) European Geriatric Medicine Society 2021

Falls are common in older adults and lead to significant morbidity and mortality. Approximately one in four older adults falls each year with $20-30 \%$ of fallers sustaining moderate-severe injuries. In addition, falls and fall-related injuries lead to excessive use of health services such as hospitalizations and emergency room visits [1].

Traditional falls prevention programs are based on identifying and modifying falls risk factors. These programs usually include: an exercise component focusing on strength and balance; a medical evaluation focusing on high-risk medical conditions, vision, orthostatic hypotension, osteoporosis, and cognition; an assessment of environmental hazards; and a comprehensive medication review [2]. While general medication reviews have not been shown to decrease falls in the long-term care setting [3], decreasing the use of psychotropics (benzodiazepines, any other hypnotic, any antidepressant or antipsychotic) was effective in reducing falls in community dwelling older adults [4].

The recently published study by Seppala et al. [5] is designed to identify fall-risk-increasing drugs (FRIDS) for possible deprescribing. This tool is meant to be used as part of a broader falls prevention strategy. They use a Delphi technique to develop the list of FRIDs-the STOPPFall (Screening Tool of Older Persons Prescriptions in older adults with high fall risk). In addition, the authors have provided practical guidance in the form of decision trees for withdrawal of drug classes used by those who have fallen, to facilitate FRID deprescribing (see appendices). The authors

Shelley A. Sternberg

sternb_sh@mac.org.il

Maccabi Healthcare Services, Modiin, Israel

2 Women's College Research Institute, Women's College Hospital, Toronto, ON, Canada

3 Departments of Medicine, Institute of Health Policy, Management and Evaluation, University of Toronto, Toronto, ON, Canada

4 Division of Geriatric Medicine and Meyers Primary Care Institute, University of Massachusetts Medical School, Worcester, MA, USA have drawn on their experience with tools that have been developed to guide appropriate prescribing in older adults (STOPP/START, FORTA, TIME, Beers) [6-9]. These tools, when incorporated into intervention studies, have been shown to decrease falls, but these tools are not consistent in their lists of FRIDs. Current European and American falls prevention guidelines do not provide a uniform list of FRIDs or provide guidance to inform deprescribing [10].

The authors' first goal in this study was to reach consensus on a comprehensive STOPPFall screening tool. The second goal was to combine the STOPPFall with practical guidance to simplify and structure FRID deprescribing. Finally, the third goal was to reach consensus and harmonize the approach to clinical management of drug-related falls across Europe. They created an initial STOPPFALL based on recent systematic reviews that examined the association between medication classes and falls risk and on eight national falls prevention guidelines. The novel element of this study was the structured Delphi process initiated with the expert panel to arrive at a consensus approach. The authors recruited 24 members of the EuGMS Task and Finish Group on FRIDs and Special Interest Group on Pharmacology representing 13 European countries, as well as an international advisory group. This group of experts, "the panelists", progressed through a series of structured Delphi rounds. They were initially asked to indicate to what extent they agreed with including a certain medication class on the list and then were asked about subclasses. To develop the deprescribing protocols, the panelists were asked to indicate whether a stepwise withdrawal was needed for every medication class, in what situations withdrawal was indicated, and how the patient should be monitored after deprescribing.

The study resulted in consensus on 14 medication classes to be included in a comprehensive list of FRIDs, STOPPFall. No consensus was reached on 17 classes. Furthermore, consensus was reached on 18 statements about the fall risk-increasing properties of subclasses within medication classes such as variation in sedative effect, degree of anticholinergic activity, and propensity to cause orthostasis. Finally, the group produced a series of decision trees based 
on prior guidance that outlined the deprescribing process for all 14 medication classes.

The authors have provided valuable tools for the practicing clinician. The STOPPFall provides an evidence and consensus-based list of 14 FRID medication classes that can be readily accessed by the health care provider. Busy clinicians need readily accessible information available on digital medication platforms linked to the medical record. STOPPFall can provide such a resource. In addition, easy access to the clear and simple deprescribing decision trees have the potential to greatly enhance and facilitate deprescribing efforts at the point of care.

A word of caution is indicated at this point. As the authors note, results of deprescribing trials for prevention of falls and fall-related injuries have been equivocal. As an example, the Strategies to Reduce Injuries and Develop confidence in Elders (STRIDE) trial published in the New England Journal of Medicine in 2020 described an extensive nurse-lead falls prevention intervention [11]. This was a pragmatic randomized trial based in 84 clinical sites in the United States. The comprehensive intervention included medication review. No statistically significant difference was found in the rate of first adjudicated serious fall injury events per 100 person-years of follow-up. The medication intervention included a review of FRIDs, drug-related side effects that may increase the risk of falls, medications without a clear indication, medication adherence problems and use of alcohol. Of note, among participants using medications that were a known risk factor for falls, only about a third agreed to address this important risk. The list of FRIDs used in this study included five drug classes of which three appear in STOPPFall.

Seppala and colleagues are to be commended for providing a useful consensus and evidence-based list of FRID (STOPPFall) classes, as well as practical deprescribing decision trees. It remains our challenge to conduct more research to determine whether using the STOPPFall tool and deprescribing high-risk drugs actually lead to a reduction in fallrelated injuries in older adults.

\section{Compliance with ethical standards}

Conflict of interest The author declares that there is no conflict of interest.

Ethical approval This article does not contain any studies with human participants or animals performed by any of the authors.
Informed consent For this type of article formal consent is not required.

\section{References}

1. Moreland B, Kakara R, Henry A (2020) Trends in nonfatal falls and fall-related injuries among adults aged $\geq 65$ yearsUnited States, 2012-2018. MMWRMorb Mortal Wkly Rep 69(27):875-881

2. Ganz DA, Latham NK (2020) Prevention of falls in communitydwelling older adults. N Engl J Med 382(8):734-743

3. Cameron ID, Dyer SM, Panagoda CE et al (2018) Interventions for preventing falls in older people in care facilities and hospitals. Cochrane Database Syst Rev 9:Cd005465

4. Gillespie LD, Robertson MC, Gillespie WJ et al (2012) Interventions for preventing falls in older people living in the community. Cochrane Database Syst Rev 9:Cd007146

5. Seppala LJ, Ryg G, Bahat G, Topinkova E, Szczerbińska K, van der Cammen TJM, Hartikainen S, Ilhan B, Landi F, Morrissey Y, Mair A, GutiérrezValencia M, EmmelotVonk MH, Caballero Mora MA, Denkinger M, Crome P, Jackson SHD, CorreaPérez A, Knol W, Soulis G, Gudmundsson A, Ziere G, Wehling M, O'Mahony D, Cherubini A, van der Velde N (2020) Stoppfall (screening tool of older persons prescriptions in older adults with high fall risk) a delphi study by the EuGMS. Age and Ageing. doi: https://doi.org/10.1093/ageing/afaa249

6. American Geriatrics Society Panel (2015) American Geriatrics Society 2015 Updated Beers Criteria for Potentially Inappropriate Medication Use in Older Adults. J Am Geriatr Soc 63(11):2227-2246

7. Bahat G, Ilhan B, Erdogan T et al (2020) Turkish inappropriate medication use in the elderly (TIME) criteria to improve prescribing in older adults: TIME-to-STOP/TIME-to-START. EurGeriatr Med 11(3):491-498

8. O'Mahony D, O'Sullivan D, Byrne S, O'Connor MN, Ryan C, Gallagher P (2015) STOPP/START criteria for potentially inappropriate prescribing in older people: version 2. Age Ageing 44(2):213-218

9. Pazan F, Wehling M (2017) TheFORTA (Fit fOR The Aged) App as a clinical tool to optimize complex medications in older people. J Am Med DirAssoc 18(10):893

10. Panel on Prevention of Falls in Older Persons (2011) Summary of the Updated American Geriatrics Society/British Geriatrics Society clinical practice guideline for prevention of falls in older persons. J Am Geriatr Soc 59(1):148-157

11. Bhasin S, Gill TM, Reuben DB et al (2020) A randomized trial of a multifactorial strategy to prevent serious fall injuries. $\mathrm{N}$ Engl $\mathrm{J}$ Med 383(2):129-140

Publisher's Note Springer Nature remains neutral with regard to jurisdictional claims in published maps and institutional affiliations. 